Die Schweiz und die humanitäre Aktion

\title{
Die Herausforderungen der Humanitären Aktion
}

Jacques Forster

\section{(2) OpenEdition}

\section{Journals}

Electronic version

URL: http://journals.openedition.org/sjep/624

DOI: $10.4000 /$ sjep.624

ISSN: 1663-9677

\section{Publisher}

Institut de hautes études internationales et du développement

\section{Printed version}

Date of publication: 1 janvier 1999

Number of pages: 3-7

ISSN: 1660-5926

\section{Electronic reference}

Jacques Forster, «Die Herausforderungen der Humanitären Aktion », Schweizerisches Jahrbuch für Entwicklungspolitik [Online], 18 | 1999, Online erschienen am: 17 Juli 2012, abgerufen am 08 September 2020. URL : http://journals.openedition.org/sjep/624 ; DOI : https://doi.org/10.4000/sjep 624

(c) The Graduate Institute 


\title{
DIE HERAUSFORDERUNGEN DER HUMANITÄREN AKTION
}

\author{
JACQUES FORSTER ${ }^{*}$
}

1

N KRISENSITUATIONEN, die durch eine Naturkatastrophe, eine technologische Katastrophe oder einen Konflikt ausgelöst werden, hat die humanitäre Aktion zum Ziel, Leben zu retten, das Leid der betroffenen Bevölkerung zu lindern und ihre Würde zu wahren. Die materielle Hilfe (medizinische Betreuung, Nahrungsmittelhilfe, usw.) ist ein wichtiger Bestandteil der Aktion, die aber nicht auf diese Dimension allein beschränkt werden darf. Der Schutz der Bevölkerung erfordert auch ein Vorgehen bei allen betroffenen Akteuren, um sie zu einem Verhalten zu bringen, das die körperliche Unversehrtheit und die Würde der von der Krise betroffenen Gruppen und Individuen achtet ${ }^{1}$.

In den neunziger Jahren hat die humanitäre Aktion in den internationalen Beziehungen viel grössere Bedeutung erlangt als in den vorangegangenen Jahrzehnten. Ein Indikator hierfür ist der Anteil der für Nothilfe aufgebrachten öffentlichen Entwicklungshilfe, der von $1 \%$ Mitte der siebziger Jahre auf $5 \%$ zwanzig Jahre später ansteigt ${ }^{2}$. Jedoch lässt sich die Bedeutung dieser Erscheinung nicht nur in Form finanzieller Ressourcen ausdrücken. Die humanitäre Aktion gehört zur Aussenpolitik zahlreicher - vor allem westlicher - Staaten und nimmt im Instrumentarium dieser Politik immer mehr Raum ein.

Könnte die zunehmende Anzahl von Katastrophen und Krisen aller Art diese Erscheinung erklären? Die Frage ist schwer zu beantworten. Vergleiche mit anderen Epochen anzustellen, wirft Probleme der Methodik auf, und sei es auch nur, weil die Information über diese Ereignisse heute viel systematischer und zugänglicher ist als vor einigen Jahrzehnten. Dennoch wird offenbar, dass die heutige Welt Spannungen unterworfen ist, welche die Risiken von Katastrophen und Krisen vermehren, deren Auswirkungen in aufeinanderfolgenden Wellen weit über ihren Ursprungsort hinaus zu spüren sind. Unter den Faktoren, welche die Weltgesellschaft anfällig machen, lassen sich folgende erwähnen :

- Ein instabiles System internationaler Beziehungen. Mit dem Ende des Kalten Krieges haben wir ein Zeitalter der Instabilität in den internationalen Beziehungen erreicht, das die Entwicklung nationaler und internationaler Krisen und Konflikte ermöglicht, welche früher durch die Spielregeln der beiden rivalisierenden Supermächte eingedämmt worden wären.

- Die Globalisierung und die Zersplitterung der Weltgesellschaft. Zwei bedeutende Kräfte gestalten diese Gesellschaft: die eine, die wirtschaftliche und technologische, treibt zu ihrer Integration, die andere drückt sich, als Reaktion, durch das Aufkommen oder Wiederaufkommen von Nationalismen, starken Gefühlen der Zugehörigkeit zu einer religiösen Bewegung, zu einer Volksgemeinschaft aus.

* Professor am IUED.

1. Dieser Artikel bezieht sich vor allem auf Konfliktsituationen; doch sind einige der angesprochenen Fragen auch für Natur- oder technologische Katastrophen relevant.

2. OCDE, Coopération pour le développement, Rapport 1997, OCDE, Paris 1998, p. A44. 
- Die Schwächung des Staates. Allgemein haben das Scheitern des kommunistischen Entwicklungsmodells, die weltweite Ausdehnung des Wirtschaftsliberalismus und die Funktionsstörungen der staatlichen Strukturen die Rolle des Staates in allen Regionen der Welt in Frage gestellt. Wenn auch in vielen Ländern eine Neudefinition der Strukturen und Funktionen der öffentlichen Hände notwendig war, ist diese jedoch oft zu weit gegangen, so dass der Staat nicht einmal mehr seine Mindestfunktionen als Ordnungshüter ausüben konnte (Bekämpfung der Kriminalität und des illegalen Handels aller Art).

- Das Aufkommen sozialer Ungleichheiten. Die Liberalisierung der Wirtschaftspolitik hat das Auftreten oder die Verstärkung der sozialen Ausgeschlossenheit besonders dort gefördert, wo die wirtschaftlichen und sozialen Strukturen den Keim einer Zweiklassengesellschaft in sich tragen. Der Staat ist wegen seiner Schwächung nicht imstande, den Zusammenhalt der Gesellschaft durch die Einführung der Korrekturmechanismen $\mathrm{zu}$ sichern, die geeignet wären, die Ausweitung des Phänomens zu vermeiden.

- Durch die Umweltbeschädigung nehmen Anzahl und Ausmass der Dürren und Überschwemmungen zu. Das Bevölkerungswachstum und der ungleiche Ressourcenzugang (Boden und Wasser) bringen Bevölkerungen dazu, sich an Orten niederzulassen, die besonders stark Naturkastrophen (Erdbeben, Vulkanausbrüchen, Überschwemmungen) ausgesetzt sind.

D IE OBENERWÄHNTEN FAKTOREN wirken zusammen und erhöhen dadurch die Risiken von Unruhen, Konflikten, Natur- und technologischen Katastrophen, deren humanitäre Folgen die lokalen Akteure nicht allein tragen können. Die Beachtung, die der humanitären Aktion heute zukommt, beruht hauptsächlich auf dem vermehrten Interesse, das sie bei den Industriestaaten erregt, wegen:

- der globalen Auswirkungen der Konflikte oder Naturkatastrophen, die dadurch, dass sie eine Region destabilisieren, die Interessen zahlreicher Staten beeinträchtigen können und ihre Wirkungen über die Region hinaus, namentlich durch grosse Bevölkerungsbewegungen verbreiten können. Die humanitäre Aktion wird als ein Mittel angesehen, das diese Auswirkungen beschränken und ihre Weiterverbreitung eindämmen kann.

- der Einbeziehung der humanitären Aktion in die Einsatzmittel der Staaten in einer Konfliktsituation, um andere politische, diplomatische oder militärische Vorgehensweisen zu ergänzen bzw. zu ersetzen.

a der Berichterstattung durch die Medien. Die heutigen Konflikte und Katastrophen werden der gesamten Bevölkerung der Industrieländer durch die Medien ausführlich zur Kenntnis gebracht, auch wenn die Berichterstattung oft selektiv, kurzlebig und auf die spektakulärsten Aspekte beschränkt ist. Die Regierungen dieser Länder werden dadurch veranlasst, aufgrund der Erwartungen ihrer öffentlichen Meinung auf die eine oder andere Weise auf diesen Schauplätzen präsent zu sein.

Dieser «zwiespältige Triumpf» der humanitären Sache ist nach François Jean: «Ergebnis der Illusion... einer in der Ablehnung des Unannehmbaren schliesslich versöhnten Menschheit und Symptom der Bestürzung angesichts des Eindrucks "neuer internationaler Unordnung". Das humanitäre Etikett wird 
seitdem so geschätzt, dass es zu einem Legitimierungspatent für jede diplomatisch-militärische Aktion, oder Scheinaktion, geworden ist $»^{3}$.

Die rasche Zunahme der staatlichen, zwischenstaatlichen und nichtstaatlichen humanitären Akteure ist auch ein Zeichen dieser Vorliebe für die humanitäre Aktion. Zwar sollten alle zur Verfügung stehenden Kompetenzen und Ressourcen mobilisiert werden können, um die Opfer von Katastrophen und Konflikten zu schützen und ihnen zu helfen, doch bleibt die Tatsache, dass diese Vielzahl von Akteuren, wegen des Mangels an Professionalismus oder Unabhängigkeit einiger von ihnen und des Wettlaufs um die Berichterstattung durch die Medien und um die Finanzmittel, der Wirksamkeit der Aktion allzu oft schaden kann.

Dsing IE NEUNZIGER JAHRE waren - leider - besonders reich an Erfahrungen hinsichtlich der internationalen Reaktionen auf akute Krisensituationen mit beträchtlichen humanitären Folgen. Von Ruanda bis Bosnien-Herzegowina, vom Sudan bis zum Kaukasus und zum Horn von Afrika haben Konflikte und Hungersnöte einen besonders hohen Zoll an Menschenleben und menschlichem Leid gefordert. Die Staaten, die internationalen Organisationen und die NRO haben immer schwierige und zuweilen traumatisierende Erfahrungen erlebt, mit sehr gegensätzlichen Ergebnissen betreffend die Möglichkeit, die Konflikte zu beenden und die Opfer zu schützen und ihnen zu helfen ${ }^{4}$. Diese Erfahrungen haben die Erwartungen bezüglich der Möglichkeiten der «militärisch-humanitären » Interventionen etwas heruntergeschraubt, wobei anzumerken ist, dass diese Interventionen stets selektiv, zwar nach den humanitären Bedürfnissen, aber auch nach den aussen- und innenpolitischen Interessen der eingreifenden Staaten beschlossen wurden.

Heute scheint der «humanitäre Interventionismus» nach einigen Enttäuschungen etwas aus der Mode gekommen zu sein und der Schwerpunkt wird mehr auf Prävention gesetzt. Dennoch bleiben die mit der humanitären Aktion zusammenhängenden Probleme weiter bestehen. In einem zunehmend komplexeren Umfeld sind die Herausforderungen, denen die humanitäre Aktion begegnen muss, zahlreich. Nachstehend sind jene Herausforderungen aufgeführt, die uns am wichtigsten erscheinen und die sich sowohl an die Regierungen als auch an die spezifisch humanitären Akteure richten.

\section{- Die Unabhängigkeit der humanitären Aktion wahren}

Anfang der neunziger Jahre treten wichtige Akteure, besonders die UNO, für die Integration der humanitären Aktion in Einsätze zur Wiederherstellung und Erhaltung des Friedens ein ${ }^{5}$. Die Erfahrungen aus mehreren Konflikten (Bosnien, Somalia, Irak) zeigen, dass dieser neue Ansatz mit der Wahrung der Unparteilichkeit der humanitären Aktion - der Bedingung für ihren Zugang zu allen Opfern - schwer vereinbar ist ${ }^{6}$. Die Aufgabe, den Frieden

3. François Jean, Le triomphe ambigu de l'aide humanitaire, Revue Tiers-Monde, avril-juin 1997, XXXVIII-2, numéro 152, pp. 641-642.

4. Bei Natur- oder technologischen Katastrophen hat die humanitäre Aktion nicht mit denselben Schwierigkeiten zu kämpfen, doch stellen sich die Probleme der Koordination und des wirksamen Mitteleinsatzes ebenfalls.

5. Boutros Boutros-Ghali, Agenda pour la paix, diplomatie préventive, rétablissement de la paix, maintien de la paix, New York, Nations Unies, 1992.

6. Jacques Forster, Raisons d'Etat et raison humanitaire, in Dérives humanitaires, Etat d'urgence et droit d'ingérence, Nouveaux Cahiers de l'IUED, Genève, PUF-IUED, 1994. 
wiederherzustellen oder zu erhalten, obliegt der Staatengemeinschaft. Die humanitäre Aktion, die nur danach strebt, die Opfer zu schützen und ihnen zu helfen, muss unabhängig sein, um mit allen Konfliktparteien «ein minimales Vertrauensklima herzustellen, ohne das der Zugang zur Bevölkerung, in einem zwangsläufig sehr gespannten Kontext, nicht aufrechterhalten werden kann »?

- Die Akzeptanz der humanitären Aktion verbessern

Die Motive und Modalitäten der humanitären Aktion werden von den Akteuren der von Konflikten oder Naturkatastrophen betroffenen Länder oft schlecht verstanden und akzeptiert. Dieser Mangel an Akzeptanz, der die Grundlage der humanitären Aktion als solche in Frage stellt, hat mehrere Ursachen. In vielen Konflikten der Gegenwart sind die Gewaltakteure vielfältig und wenig strukturiert. In den geschwächten Staaten sind es «Banden, Milizen, ja sogar "Sonntagskämpfer" ohne Ideologie und Programm, für die Schiessen ein Selbstzweck und das Gewehr ein Mittel zur Bestreitung des Lebensunterhalts ist ${ }^{8}$. In diesem Zusammenhang ist es nicht erstaunlich, dass die Motivationen und die Grundsätze der humanitären Aktion schlecht bekannt sind und missverstanden werden. Eine gründliche Kenntnis des Milieus und eine ständige Bemühung zur Anpassung der humanitären Botschaften an die lokalen Gegebenheiten sind eine notwendige Bedingung für die Verbesserung dieser Akzeptanz. Genereller finden die Konflikte und Katastrophen heute fast ausschliesslich in den Entwicklungsländern statt, wogegen die internationalen humanitären Akteure vor allem aus den Industrieländern kommen. Daher kann die humanitäre Aktion als ein Instrument der Industrieländer angesehen werden und aus diesem Grunde Abwehrreaktionen hervorrufen.

\section{- Die Koordination der humanitären Akteure stärken}

Die Vielzahl humanitärer Akteure vor Ort wirft Probleme auf wegen der Vielfalt und Verschiedenartigkeit der Lösungen zur Deckung der humanitären Bedürfnisse und der Grundsätze der humanitären Aktion. Hieraus können sich Inkohärenzen und eine Ressourcenverschwendung ergeben, die dem Image der humanitären Aktion bei den Opfern, den Protagonisten und der breiten Öffentlichkeit erheblich schaden. Deshalb müssen die Geldgeber und die humanitären Akteure Verhaltensregeln unterstützen und anwenden, welche die Unabhängigkeit, die Unparteilichkeit und den Professionalismus der humanitären Aktion gewährleisten? .

- Die humanitäre Aktion

mit der Entwicklung abstimmen und in die lokale Realität verankern

In Notsituationen haben Interventionen von aussen Tendenz, den zur Rettung von Leben dienenden sofortigen Bedürfnissen nachzukommen, ohne sich allzu sehr um die lokalen Ressourcen und Fähigkeiten zu kümmern. Jedoch erstreckt sich die humanitäre Aktion in vielen Krisensituationen über lange Zeiträume (Jahre, bzw. Jahrzehnte) hinweg. Die Dichotomie «Notstand - Entwicklung» verliert dann an Relevanz und die humanitäre Aktion muss Modalitäten einführen, welche die Perspektive der langfristigen Entwicklung berücksichtigen.

7. François Jean, op.cit., p. 646.

8. Jean-Pierre Langellier, Le Monde, 18 juin 1997.

9. 1995 haben 142 Regierungen dem Verhaltenskodex für die Internationale Bewegung des Roten Kreuzes und Roten Halbmonds und für die NRO bei Hilfsoperationen im Katastrophenfall ihre Unterstützung gewährt. 
Allzu oft werden die lokalen Mechanismen und Institutionen durch die Intervention von aussen ignoriert. Deshalb liegt die Herausforderung darin, so früh wie möglich die Akteure der Zivilgesellschaft mit einzubeziehen, die beim wirtschaftlichen, politischen und sozialen Wiederaufbau die wesentliche Rolle spielen werden.

\section{- Die Wirksamkeit der humanitären Atkion verbessern}

In einer Zeit, die durch Haushaltskürzungen und die Sorge gekennzeichnet ist, die öffentlichen Finanzmittel effizienter zu verwalten, nimmt die Frage der Wirksamkeit und der Effizienz (Verhältnis zwischen Resultaten und Kosten) der humanitären Hilfe im Dialog zwischen den Geldgeberstaaten und den humanitären Institutionen immer mehr Raum ein. Die Praxis gründlicher Evaluationen verbreitet $\operatorname{sich}^{10}$ und die Erwartungen der Geldgeber in Bezug auf Informationen über die Durchführungsverfahren und -kosten der humanitären Organisationen steigen beträchtlich. Ihrerseits müssen die Geldgeber auch - um der Kohärenz willen - mehr Flexibilität bei den Bedingungen hinsichtlich der Verwendung der Ressourcen zeigen, die sie den humanitären Organisationen zur Verfügung stellen.

\section{- Die Kohärenz der Politiken stärken}

Seit einigen Jahren sind sich die Regierungen der Industrieländer der engen Beziehungen zwischen den verschiedenen Bereichen ihrer Politik gegenüber den Entwicklungsländern bewusst geworden. Unter dem Titel «Kohärenz der Politiken» werden derzeit in mehreren Ländern Anstrengungen dahingehend unternommen, dass alle Politiken zur Verwirklichung des Ziels der Förderung der wirtschaftlichen und sozialen Entwicklung dieser Länder beitragen ${ }^{11}$. Aus dieser Sicht heraus ist es wichtig, dass die Handelsbeziehungen (einschliesslich Waffentransfers), die ausländischen Investitionen und die Hilfe, besonders im Bereich der sozialen Entwicklung und der Verstärkung der öffentlichen Einrichtungen, dazu beitragen, die zu Beginn dieses Artikels erwähnten Spannungsfaktoren $\mathrm{zu}$ reduzieren (oder zumindest nicht zu erhöhen). Denn die grösste Herausforderung aller, die sich für die Entwicklung und die humanitäre Aktion engagieren, ist es doch, eine im Süden und im Norden abgestimmte Aktion durchzuführen, um die Konflikte zu verhüten.

10. Für Afrika wurden zwischen 1994 und 1996 zahlreiche ausführliche Evaluationen von Regierungen und grossen internationalen Organisationen durchgeführt. Sie werden analysiert in Raymond Apthorpe, Some Relief from Development: Humanitarian Emergency Aid in the Horn of Africa (including Sudan), Rwanda and Liberia, The European Journal of Development Research, Vol. 9, Number 2, December 1997, Frank Cass, London.

11. In der Schweiz wird diese Politik im «Leitbild Nord-Süd» angekündigt, das vom Bundesrat im März 1994 verabschiedet wurde. 